\title{
TCP- Costco Reno: New Variant by Improving Bandwidth Estimation to adapt over MANETs
}

\author{
Prakash B. Khelage \\ Asst. Professor, Information Technology, \\ UMIT, SNDT Women's University, Mumbai-400049, \\ India.
}

\begin{abstract}
The Transmission Control Protocol (TCP) is traditional, dominant and has been de facto standard protocol, used as transport agent at transport layer of TCP/IP protocol suite. Basically it is designed to provide reliability and assure guaranty to end-to-end delivery of data over unreliable networks. In practice, most TCP deployments have been carefully designed in the context of wired networks. Ignoring the properties of wireless Ad Hoc Networks, therefore it can lead to TCP implementations with poor performance. The problem of TCP and all its existing variations within MANETs resides in its inability to distinguish between different data packet loss causes, whenever the data loss occur traditional TCP congestion control algorithm assumes loss is due to congestion episode and reduces sending parameters value unnecessary. Thus, TCP has not always the optimum behavior in front of packet losses which might cause network performance degradation and resources waste. In order to adapt TCP over mobile Ad hoc environment, improvements have been proposed based on RTT and BW estimation technique in the literature to help TCP to differentiate accurate causes between the different types of losses. But still does not handle all the problems accurately and effectively. In this paper, a proposed TCP-Costco Reno a New Variant, accurately estimates the available bandwidth over Mobile Ad Hoc networks and sets sending rate accordingly to maximize utilization of available resources and hence improves performance of TCP over mobile Ad hoc networks. The results of the simulation indicate an improvement in throughput over interference, link failure and signal loss validation scenarios. Further, it shows highest average of average throughput then those variants which are most successful over MANETs.
\end{abstract}

Keywords-mobile ad hoc network (MANET); Ccongestionl; Link failure;signal loss; interference; Retransmission timeout; RTT and $B W$ estimation

\section{INTRODUCTION}

The phenomenal growth experienced by the Internet over the last decade has been supported by a wide variety of evolving mechanisms to meet the requirements of emerging, demanding applications. The basic TCP/IP protocol suite has been instrumental in developing today's Internet. In particular, TCP has been successful due to its robustness in reacting dynamically to changing network traffic conditions and providing reliability on an end-to-end basis. This Wide acceptance has driven the development of many TCP applications, motivating the extension of this protocol to wireless networks. These networks pose some critical challenges to TCP since it was not originally designed to work in such complex environments, where the level of bit error rate (BER) is not negligible due to the physical medium[3][9].

\author{
Dr. Uttam D. Kolekar \\ Principal Smt. Indira Gandhi College of Engineering, \\ Navi Mumbai - 400709, \\ India.
}

High mobility may further degrade the end-to-end performance because TCP reduces its transmission rate whenever it perceives a dropped packet.

Mobile ad hoc network is a collection of mobile nodes that offers different opportunities to TCP. Reduction in deployment cost due to absence of fixed infrastructure and elimination of administration cost since it is self-configurable.

However, MANET consists of unstable wireless communication links in compare to the wired network [1]. This instability is mainly due to mobility of nodes. Because TCP is originally invented for wired network [3], it ignores non-congestion loss which occurs rarely in this environment. Thus, TCP in present form cannot address frequent link breakage in MANET and suffers from performance degradation [4]. TCP is responsible for providing reliability of connection by retransmitting lost packet. Congestion control is the most controversial parts of TCP which degrades performance in front of packet loss [9]. Congestion control as its name appears, assumes all packet loss induced by congestion.

When link failure lasts greater than RTO (Retransmission timeout), Retransmission timer expires and TCP interprets packet loss as a congestion loss. Then congestion control executes back-off algorithm to grow RTO exponentially and retransmit packet. After a few successive back-off executions, RTO becomes too long. Hence when route recovered, sender resumes data transmission with long RTO which forces sender remains idle unnecessary in case of probable next losses[2]. Thus, traditional TCP fails in wireless network. As per the literature study, the proposals based on RTT (retransmission time) and BW (Bandwidth) estimation technique are successful till somewhat extent and not utilizing available resources optimized. Therefore further improvement or modification need to be done. This paper focused on the modification of TCP-Westwood which is sender side modification and based on BW (bandwidth) estimation Technique.

The remainder of the paper is organized as follows. In Section II, literature survey of TCP-Westwood and TCPWELCOME with its algorithms, problems and comparative analysis is presented. Section III defines the problems with existing Solution. Section IV explains about the proposal for solution. Tools and Techniques, Basic validation Scenario with discussion and experimental results presented in section V. Section VI Acknowledges to those who motivated and 
helped me lot for doing this research work. Section VII concludes the paper.

\section{LITERATURE SURVEY}

\section{A. TCP Westwood}

TCP Westwood proposes an end-to-end bandwidth estimation algorithm based on TCP Reno. TCP Westwood implements slow start and congestion avoidance phases as TCP Reno, but instead of halving the congestion window size as in TCP Reno when congestion happens, TCP Westwood adaptively estimates the available bandwidth and sets the congestion window size and slow start threshold accordingly to improve the link utilization. In TCP Westwood, packet loss is indicated by the reception of 3 duplicated acknowledgements (DUPACKs) or timeout expiration. When 3 DUPACKs are received, TCP Westwood sets SSThreshHold and CWND as follows:

if (3 DUPACKs are received)

SSThreshHold $=(B E * R T T$ min $) /$ seg_size;

if $(C W N D>$ SSThreshHold) $/ *$ in congestion avoidance phase*/

CWND = SSThreshHold;

endif

endif

Where the seg_size is the length of the TCP segments and RTTmin is the minimum RTT experienced. BE is the estimated available bandwidth. It is assumed in TCP Westwood that when 3 DUPACKs are received in the congestion avoidance phase, the available bandwidth is fully utilized. So the values SSThreshHold and CWND should reflect the estimated bandwidth $(\mathrm{BE})$. If a packet loss is indicated by timeout expiration, TCP Westwood sets SSThreshHold and CWND as follows:

If (timeout expires)

$C W N D=1$;

SSThreshHold $=(B E * R T T$ min $) /$ seg_size;

if (SSThreshHold $<2)$

SSThreshHold = 2;

endif

endif

This sets the CWND to 1 and SSThreshHold to BE after the timeout event and then the TCP Reno behavior continues. In TCP Westwood, the setting of SSThreshHold and CWND is based on the bandwidth estimation, which is obtained by measuring the rate of the acknowledgments and collecting the information of the amount of packets delivered to the receiver in the ACK. Samples of bandwidth are computed as the amount of packet delivered divided by the inter-arrival time between two ACKs. Those sample bandwidth estimates are then filtered to achieve an accurate and fair estimation.
TCP Westwood modifies the Additive Increase and Multiplicative Decrease (AIMD) in TCP Reno and adaptively sets the transmission rates to remove the oscillatory behavior of TCP Reno and to maximize the link utilizations. But this also causes TCP Westwood to degrade the performance of TCP Reno connections when they coexist in the network [11].

\section{Problems:}

The behavior of the bandwidth estimation scheme is unpredictable, therefore TCP Westwood Perform poorly if it estimates incorrect bandwidth. Changes in the inter-arrival times of the acknowledgements cause improvement or worsening of the throughput in rather unpredictable ways. Additionally, the sensitivity of TCP Westwood Acknowledged Interval is variable.

\section{B. TCP WELCOME}

It is a sender-based solution, known as TCP-WELCOME, to improve the TCP performance for route failure, wireless error and congestion losses in MANET based on RTT. TCPWELCOME distinguishes between causes of packet loss and then triggers the most appropriate packet loss recovery according to the identified loss cause. It realizes its loss differentiation by observing the history of RTT samples evolution over the connection and the data packet loss triggers (3DuplACK and RTO). If loss is detected by 3DuplACK and RTT values are stable then it is wireless related packet loss or else it is congestion. On the other hand, if loss is detected by RTO and RTT values are stable then it is route failure related loss otherwise, it is congestion [4].

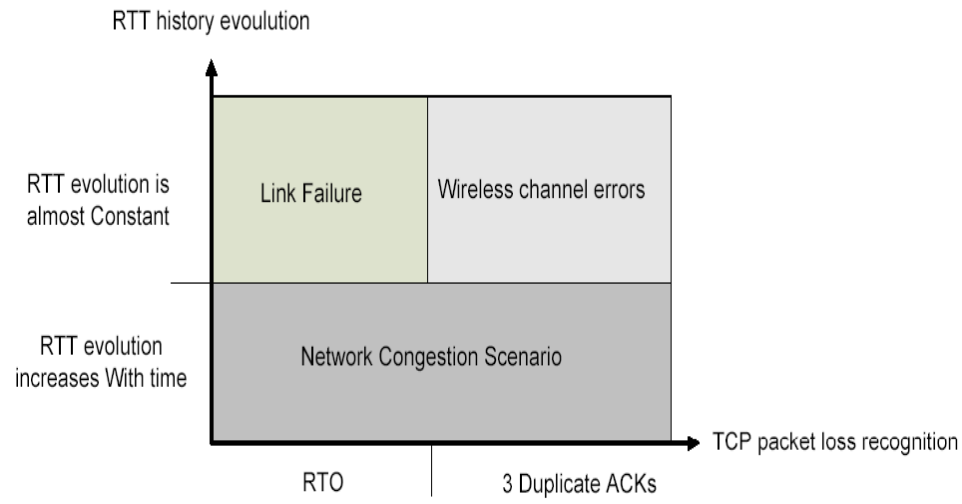

Fig. 1. TCP WELCOME Loss different algorithm [4]

Fig. 4 summarizes the main idea of loss differentiation Algorithm. After identifying the cause of a data packet loss using the proposed LDA, TCP-WELCOME react will concern on RTO calculation and data transmission rate. So in case of congestion loss no change is required to the standard TCP New Reno, the same goes for wireless error no change only retransmit the lost packet, however, once route failure is detected the RTO value and CWND would be updated based on the new route characteristics( length, load and quality) as follows:

RTO new $=($ RTTnew $/$ RTTold $) \times$ RTOold

$C W N D$ new $=($ RTTold/RTTnew $) \times C W N D$ old 


\section{Problems:}

Does not take network disconnection and frequent route change affecting into account during the evaluation, WELCOME uses RTT which include both delays of forward and reverse path while only delay of forward path must be considered. In addition, it offers recovery method based on RTT Comparison TCP-Welcome claimed that RTO adjustment should be done based on the capabilities of discovered route such as length; load and link quality [6] after link breakage, total delay for new route varies from broken route.

TABLE I. COMPARATIVE ANALYSIS OF TCP- VARIANTS

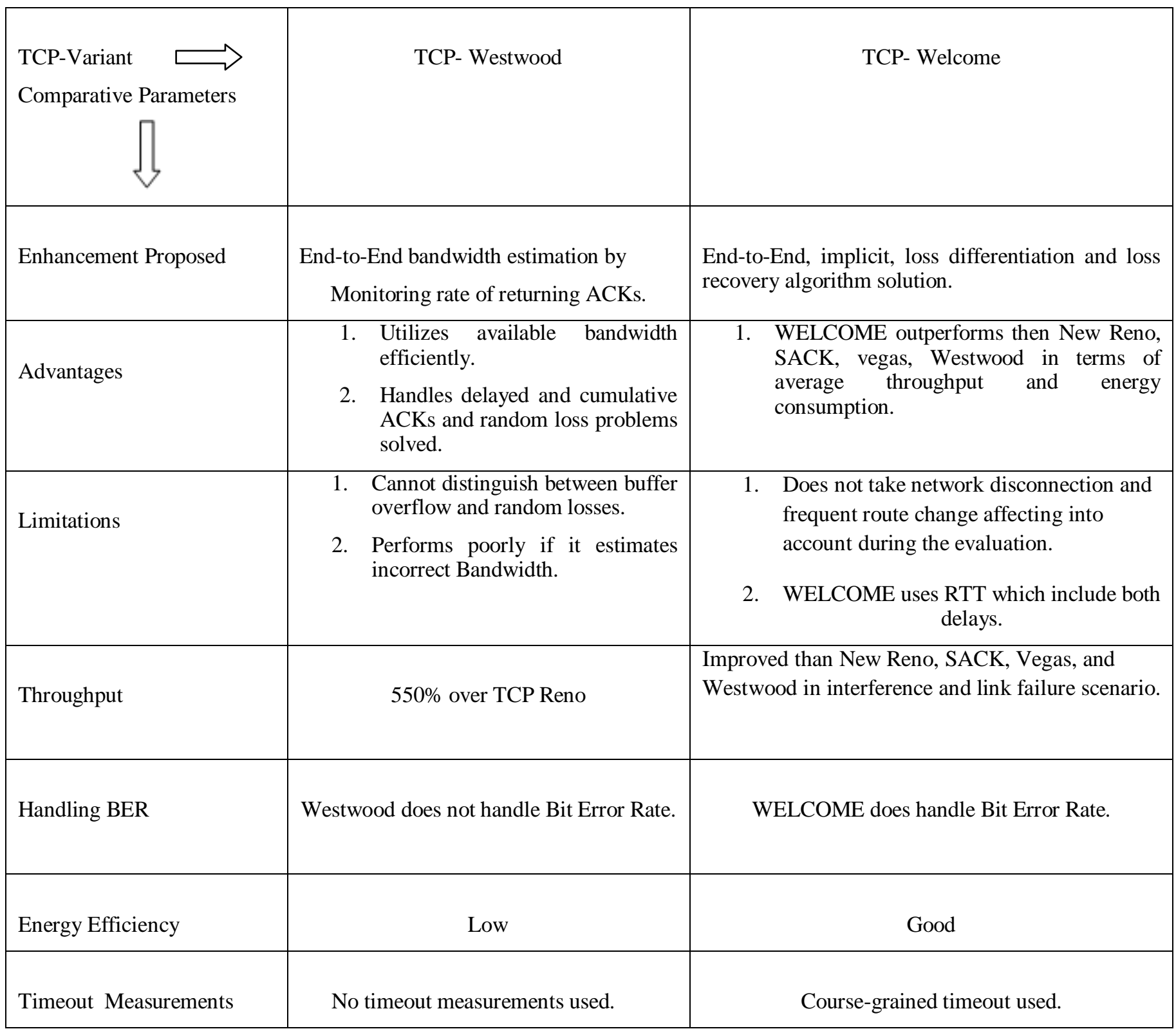

\section{PROBLEM DEFINATION}

In ad hoc networks, the principal problem of TCP lies in performing congestion control in case of losses that are not induced by network congestion. Since bit error rates are very low in wired networks, nearly all TCP Variants assume that packets losses are due to congestion. Consequently, when a packet is detected to be lost, either by timeout or by multiple duplicated ACKs, TCP slows down the sending rate by adjusting its congestion window. Unfortunately, wireless networks suffer from several types of losses that are not related to congestion, making TCP not adapted to this environment. With the help of Literature survey of TCP over Ad Hoc Networks, identified following problems. In Wireless ad-hoc networks nodes may be mobile therefore no predefined topology.

As nodes can join and leaves network, so accordingly topology may change. When the topology of the network changes every time, then routing mechanism needs to trigger 
to find alternative roots to do the reliable end to end communication between sender and receiver.

Thus due to frequently changes in topology communication links may failures and there will be the loss of data segment. To recover segment loss TCP sender reduces sending rate by triggering congestion control mechanism which sets size of congestion window of its lowest value. Assuming that the loss of packet is due to congestion in network which is totally misjudged and there will be the underutilization of available bandwidth. Hence the performance of TCP degrades. These problems are due to lossy channels, Hidden and exposed stations, path asymmetry which may appear in several forms like BW asymmetry, loss rate asymmetry and route asymmetry.

A large number of approaches for RTT and BW estimation proposed but none of them work well in all scenarios without any drawback or side effect. So it is essential that an improved BW estimation technique is evolved. The focus of our research is to study the existing techniques, propose an improved $\mathrm{BW}$ estimation technique based on TCP-Westwood which will have to reduce under and over estimation of available bandwidth before transmission.

\section{PROPOSAL FOR SOLUTION}

\section{A. Introduction}

TCP variants are well adapted to deal with all data packet loss situations that can be encountered within wireless ad hoc networks. The performance of TCP degrades significantly within wireless ad hoc networks [8]. Moreover, some of the studied TCP variants perform well in certain cases while they perform badly in other cases. The ability of TCP to distinguish among congestion-induced and wireless-related data losses (as in TCP Westwood) leads to an improved performance in some cases. However, TCP variants that incorporate a loss differentiation algorithm do not consider all types of data packet loss that can be encountered within wireless ad hoc network environments. In fact, they consider congestioninduced and wireless-channel related losses only. It also finds that the TCP variant which is able to adjust its performance parameters (CWND and RTO) after data losses (as in TCP Vegas) [5] [6], in certain cases, can improve the performance within the network.

In this paper, we propose TCP-Costco Reno a new TCP variant that is based on the modification of Westwood, which will be able to deal effectively with the under as well as over estimation of available bandwidth.

\section{B. Motivation}

The main motivation behind this research work is TCP Westwood and WELCOME Variants. Which are most successful in wireless Ad hoc network but TCP Westwood does wrong estimations, like over or under estimation of the available bandwidth due to variable delay in returning ACK Whereas, TCP WELCOME does not handle network disconnection and frequent route change. The research work has been concentrated on bandwidth estimation based technique.

\section{Problems with existing solution}

TCP Westwood is designed to perform well in wired, wireless and mixed networks. In TCP Westwood the TCP Reno congestion control is used and is modified only on the sender side [10]. TCP Westwood defines that if the congestion window size divided by the minimum RTT is larger than the currently achieved rate the channel is congested and if it is equal to the current rate, the loss is of random nature. The key innovative idea behind TCP Westwood is that it takes advantage of an end-to-end bandwidth estimation mechanism called Bandwidth Share Estimates (BSE) to set the values of slow start threshold (SSThreshHold) and congestion window (CWND) after a random (due to the lossy nature of a wireless link) loss (indicated by 3 duplicated acknowledgements that have been received or by a specific timeout).

Instead of setting the slow start threshold after receiving 3 duplicated ACKs or after the timeout expires to half of the size of the congestion window (as in TCP Reno) TCP Westwood sets the SSThreshHold to the product of the BSE and the minimum RTT. This estimation is not based on measurements performed by lower layers and therefore retains to layer principles like separation and modularity of layers. The bandwidth is estimated by the source that monitors continuously the received TCP acknowledgements. It then estimates the data rate currently achieved by the connection. By doing this, TCP Westwood ensures both faster recovery and more effective congestion avoidance. However TCPWestwood cannot distinguish between buffer overflow and random losses, Performs poorly if it estimates incorrect Bandwidth and it is not sufficiently evaluated. Therefore the mechanism of TCP Westwood modified and named as TCP Costco Reno, as this variant also retains the principle of New Reno.

\section{TCP-Costco Reno (New Variant for MANET)}

As per the pseudo code of TCP Westwood, it has calculated the value of SSThreshHold and compared it with CWND and then according to it sets the value of CWND. It is noticed that, it should not forcefully increase CWND if it is smaller than SSThreshHold, as well as this mechanism can't set the value properly, Hence Bandwidth estimation is inaccurate in case of TCP-Westwood, which faces problem like over and under estimation of available bandwidth over wireless networks scenarios.

Over bandwidth estimation: to set bandwidth more than available bandwidth.

Under bandwidth estimation: to set bandwidth less than available bandwidth.

So, to overcome above problems the proposal estimates bandwidth properly by using following code:

Set SSThreshHold $=(\min 4$ or maximum calculated value $)$

Set slow start $=2$

thresh_= $($ int $)(($ current_bwe_/size_/8)*in_rtt_estimate $)$;

if (thresh_> 4)

SSThreshHold_ $=$ thresh_ 


$$
\text { else }
$$

if (thresh_<4)

$$
\begin{aligned}
& \text { SSThreshHold_ }=4 \\
& \text { if (CWND_> SSThreshHold_) } \\
& \text { CWND_= SSThreshHold_ }
\end{aligned}
$$

Thus, TCP Costco Reno sets optimum value of bandwidth and hence it recovers problems of under and over estimation.

In wireless environment whenever packet lost happens traditional TCP assumes that, it is due to congestion, but packet lost in wireless environment may happens due to congestion, link failure or wireless channel errors. All the times packet loss is may not due to congestion, so TCP Variant has to consider link failure and wireless channel errors also. Every time when it considers packet lost is due to congestion then TCP Westwood sets slowstart as "1" but in case of other Scenario like link failure and wireless channels errors no need to set slowstart as 1 all the time. So this is the reason that, in TCP Costco Reno sets slowstart equal to 2.

\section{E. Pseudo code of TCP-Costco Reno}

A. Algorithm after 3 duplicate ACKS:

If(3 dupack received)

thresh_= (int $)(($ current_bwe_/size_/8)*in_rtt_estimate $)$;

if (thresh_> 4

SSThreshHold_ $=$ thresh_

else

if (thresh_<4)

SSThreshHold_ $=4$

if (CWND_> SSThreshHold_)

CWND_ = SSThreshHold_

B. Algorithm after slowstart

if $\left(C W N D_{-}<S S T h r e s h H o l d \_\right)$

slowstart $=2$

\section{Tools, Validation Model And Simulation ENVIRONMENT}

NS-2 is a discrete event simulator written in $\mathrm{C}++$, with an OTcl interpreter shell as the user interface that allows the input model files (Tcl scripts) to be executed. Most network elements in NS-2 are developed as classes, in object-oriented fashion [7]. NS2 provides substantial support for simulation of TCP, routing algorithms, queuing algorithms, and multicast protocols over wired and wireless (local and satellite) networks, etc.
It is freely distributed. So, in order to investigate and understand the behavior of the congestion scheme and to observe the improved performance of our approaches in our research work we are using NS-2 as network simulator tool. We have planned to compare it with different TCP variants using different simulation scenarios with different proactive and reactive routing protocols that will describe multiple data packet loss causes which are related to wireless ad hoc networks.

We have used NS2 as a network simulation tool for hypothesis testing and Study the effect of the different loss scenarios (link failure, congestion, signal loss and interference) Evaluation of TCP-Costco Reno and comparisons with other TCP variants such as Tahoe, Reno, New-Reno, Vegas, Sack and Westwood.

In our simulation experiments we used proactive and reactive routing protocol and investigate which protocol is suitable for wireless ad hoc network to adapting TCP over ad hoc network. Nodes communicate through identical wireless radio settings using the standard MAC 802.11.

TABLE II. SimUlation PARAMETERS VALUES

\begin{tabular}{|l|l|}
\hline Parameter & Values \\
\hline Channel Type & Wireless channel \\
\hline Radio Propagation Model & Two Ray ground \\
\hline Queue type & Droptail/PriQue \\
\hline Max. packet(buffer size) & 50 \\
\hline Network interface & Wirelessphy \\
\hline MAC Protocol & 802.11 \\
\hline Data Rate & $1 \mathrm{Mbps}$ \\
\hline Transmission Radius & 250 \\
\hline Interference Radius & 550 \\
\hline Packet size & 1000 bytes \\
\hline Routing protocol & AODV, DSDV \\
\hline Simulation Time & $150 \mathrm{~s}$ \\
\hline Value x & 700 \\
\hline Value y & 500 \\
\hline Agent trace & ON \\
\hline Mac trace & ON \\
\hline Router trace & \\
\hline Movement trace & \\
\hline
\end{tabular}




\section{A. Basic Validation scenarios}

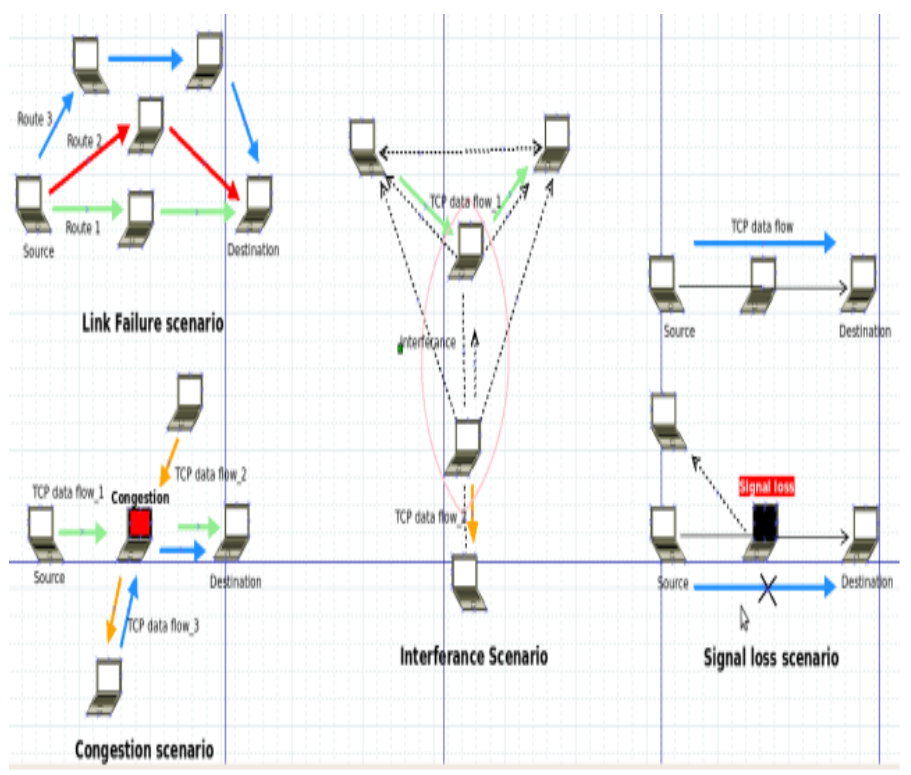

Fig. 2. basic Validation Scenario

The basic validation scenarios, using NS-2, are implemented as follows:

1) Congestion validation scenario: In this scenario, congestion created at the middle of a five node topology by generating three TCP data traffic flows that must pass by intermediate node to reach the other communicating end point. Different levels of data congestion generated by controlling the number of TCP data flows crossing intermediate node at a certain time. Congestion scenario created sending multiple TCP sources through a bottleneck link. In congestion also Costco Reno provides best average throughput than new Reno, Vegas, sack and nearly equal to Westwood.

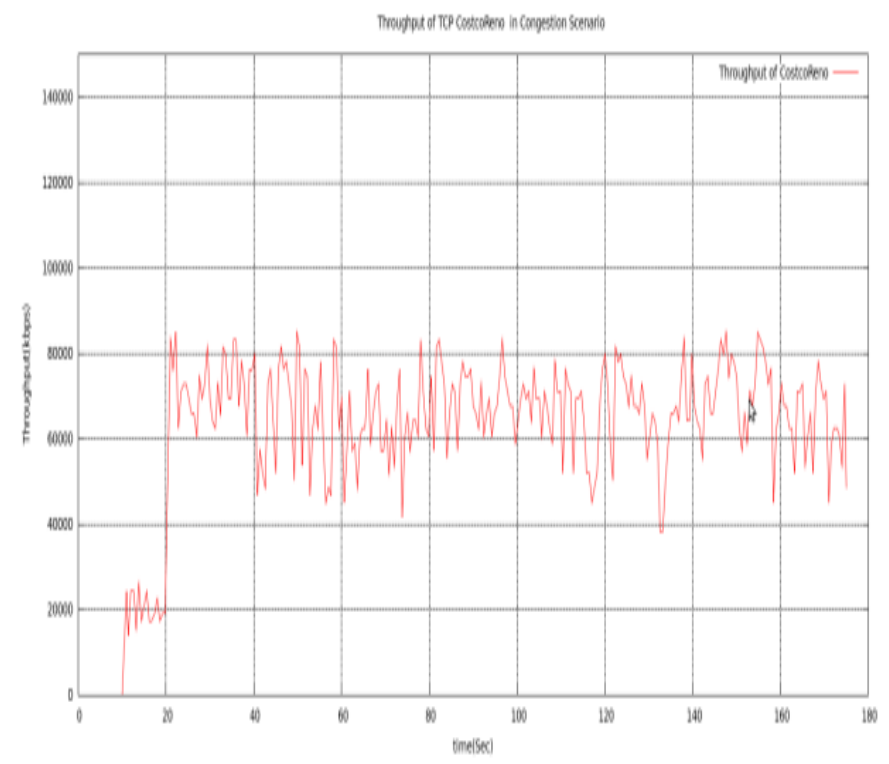

Fig. 3. Throughput of Costco Reno in congestion

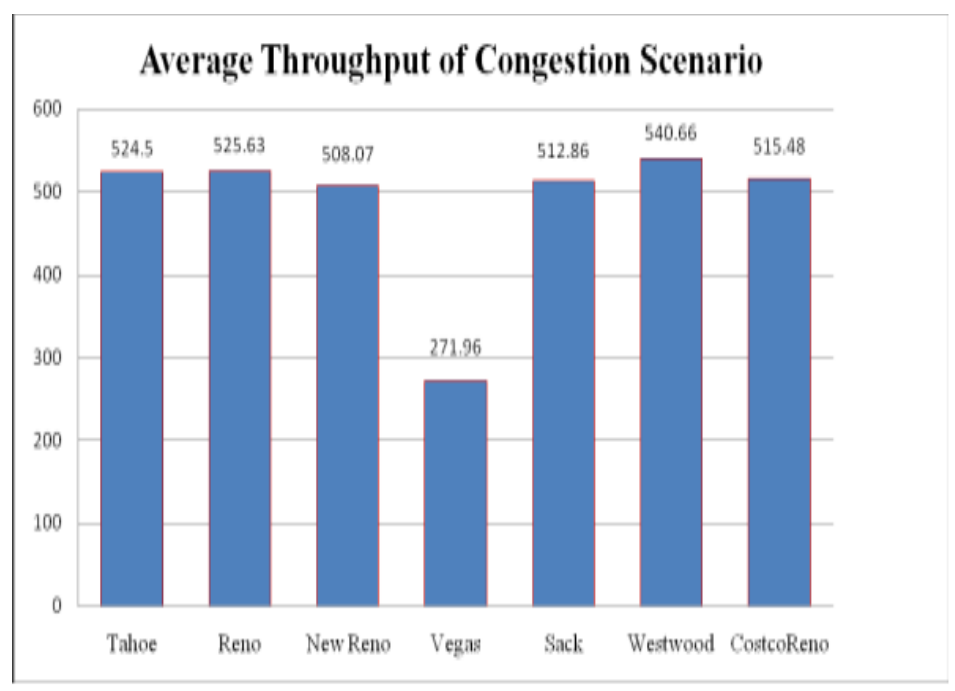

Fig. 4. average throughput of all TCP variants in congestion s scenario

2) Interference Validation Scenario: In this case, two TCP connections are established in parallel. The main TCP connection (TCP data flow 1 in Figure 6) is disturbed by the interferences generated by the second TCP connection. Indeed, the node acting as forwarder for the main TCP connection is placed within the interference range of the second TCP connection sender. So, this situation creates interference and thus data packet losses. In this scenario also TCP Costco Reno gives better average throughput than Westwood and all other TCP variants except sack by 0.15 .

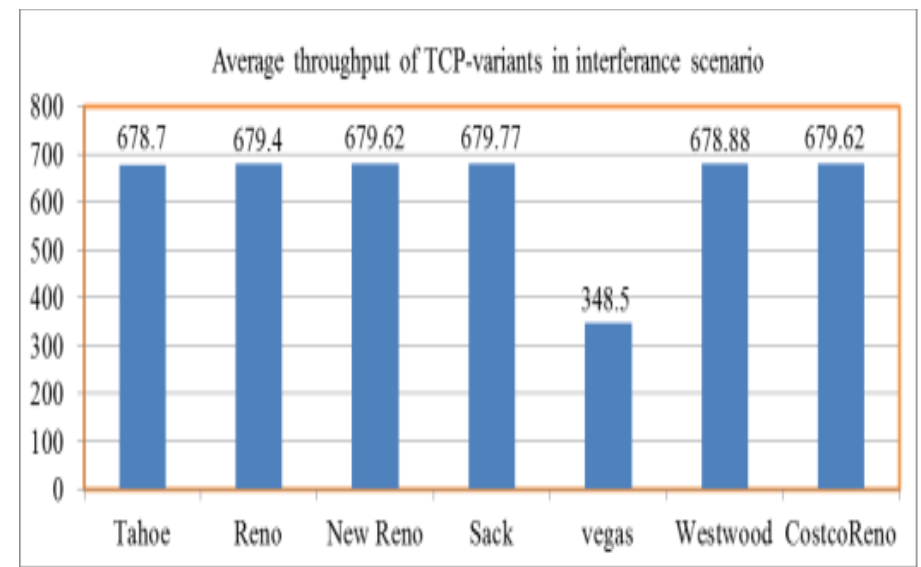

Fig. 5. Average throughput of all TCP variants in Interference scenario

3) Link Failure Validation Scenario: In link failure validation model it has been forced to TCP traffic to change its communication path by shutting down the intermediate node between the communicating ends nodes. In addition, it employs routes with different number of hops. Thus, each time TCP changes the communication route, the characteristics of the path between the communicating nodes changes. It is obvious that the choice and the establishment delay of the new route will be dependent on the implemented ad hoc routing protocol. Packet losses and delay changes will also be generated by the link loss and the new chosen route. 
Link failure scenario is created by five nodes, source and destination provided with mobility. Due to mobility both sender and receiver will be out of transmission and reception range link goes down for few seconds and few packets will drop. Other node join the mobile ad hoc network, provides transmission and reception range to sender and receiver node again transmission starts through new link. Referring with fig. 6 in this scenario also TCP-Costco Reno gives better performance than all other TCP-variants.

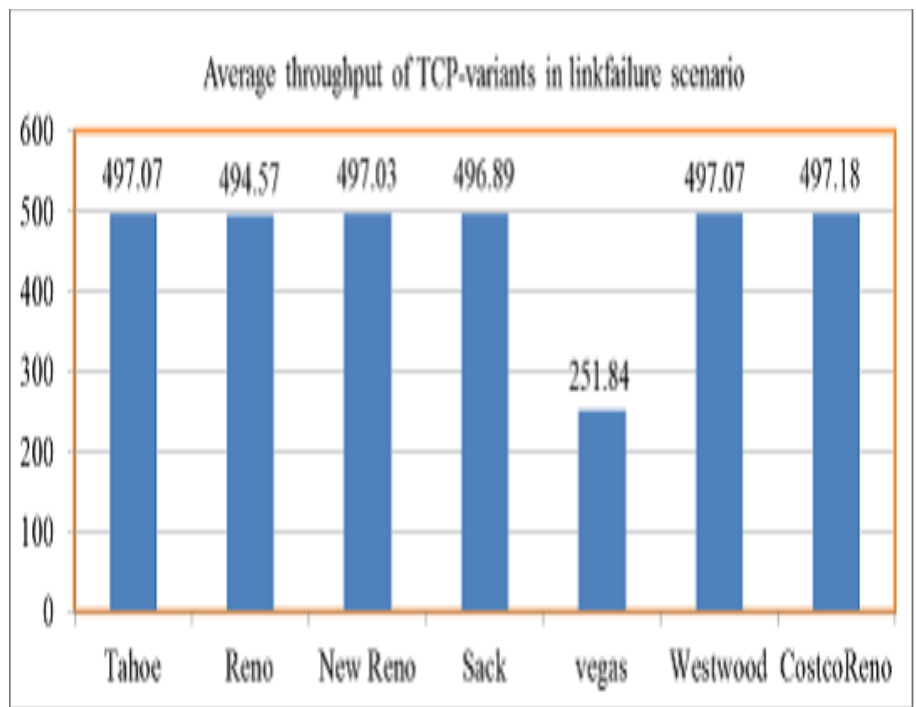

Fig. 6. Average throughput of all TCP variants in link failure scenario

4) Signal loss Validation scenario: This scenario illustrates the situation where the wireless signal is not stable. The communicating nodes loose the connection due to signal loss then they resume the communication when the signal comes back. Signal losses are generated by moving one of the intermediate nodes out of the radio range of its connection neighbours. This scenario created using three nodes. End nodes acts as sender and receiver and intermediate node as router Transmission of ftp traffic source flow through intermediate node. Intermediate node moves away for few second so signal loss occurs between source and destination, after few second intermediate node moves at original place and again retransmission starts. In signal loss scenario TCP-Costco Reno performs better than all other TCP variant as shown in figure: 7

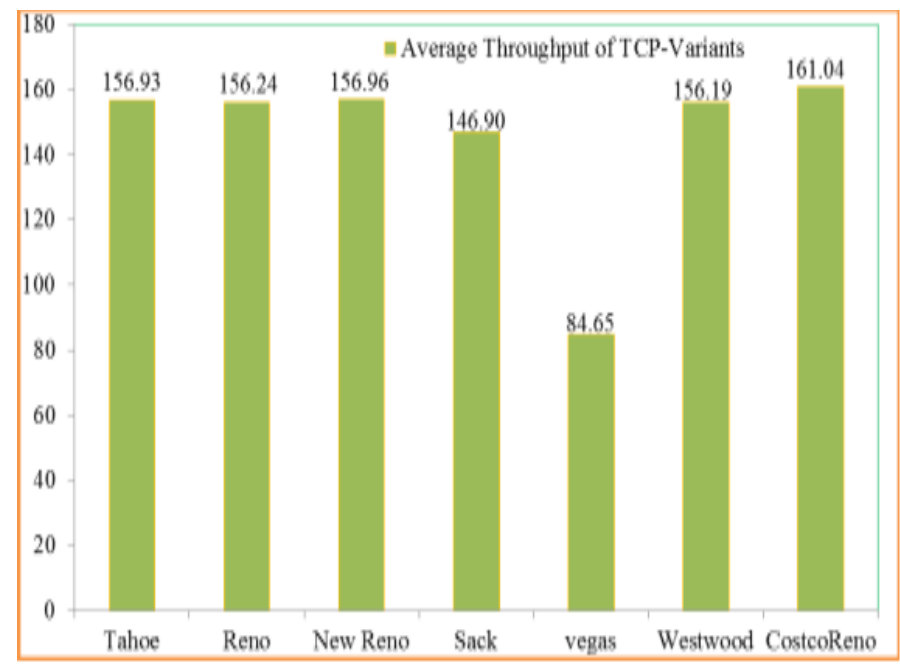

Fig. 7. Average throughput of all TCP variants in signal loss scenario

\section{B. Main Simulation scenarios Chain multi-hop network:-}

The network consists of variable length chain of static nodes, placed at a distance of $200 \mathrm{~m}$ from one another. FTP traffic is transferred between the first and last node of the chain shown network.

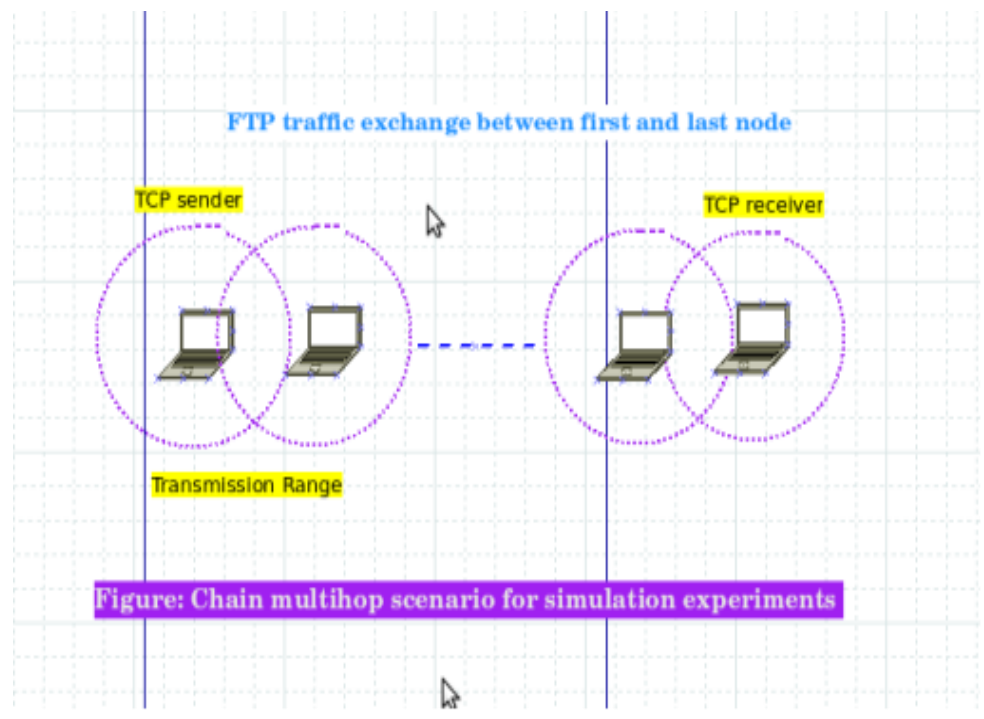

Fig. 8. Chain Topology 
During the simulation one FTP connection kept active at a time. Sequential TCP connection are initiated and terminated. The TCP-Costco Reno used at the transport layer which is an improvement of TCP Westwood. For the simulation of chain topology three hop networks created consisting of four nodes whereas, end nodes acts as sender and receiver. In this case TCP Costco Reno performs same as Tahoe as well as Tahoe based other variant but very much better then Vegas.

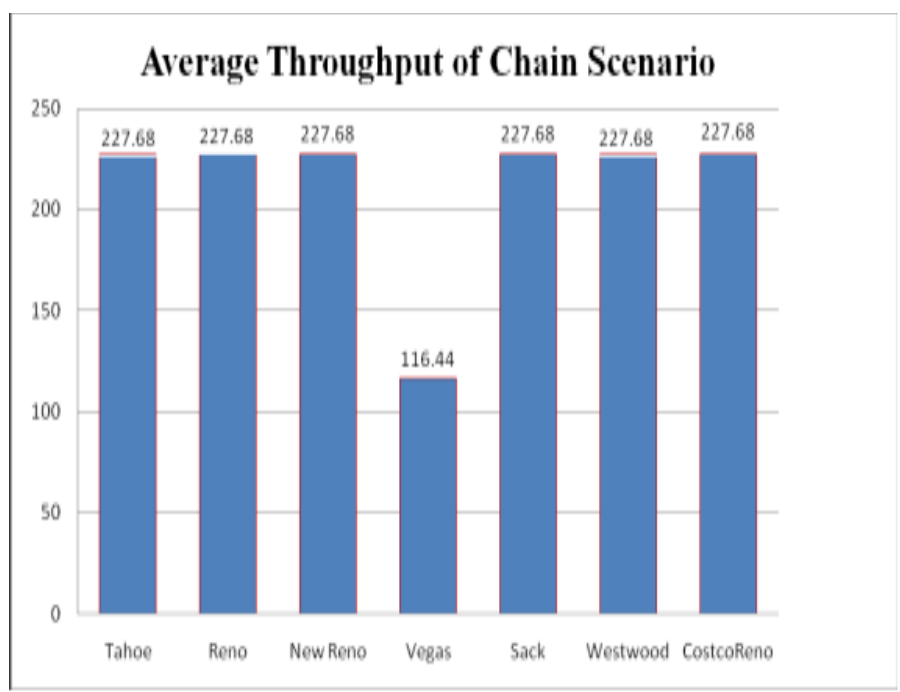

Fig. 9. Average throughput of all TCP variants in Chain Topology

\section{Grid network:-}

Fig. 10 shows a static grid network as experiment topology with $3 X 4$ nodes. The distance between two adjacent nodes is set to be $150 \mathrm{~m}$, and the transmission and interference radii are set to 250 and $550 \mathrm{~m}$, respectively.

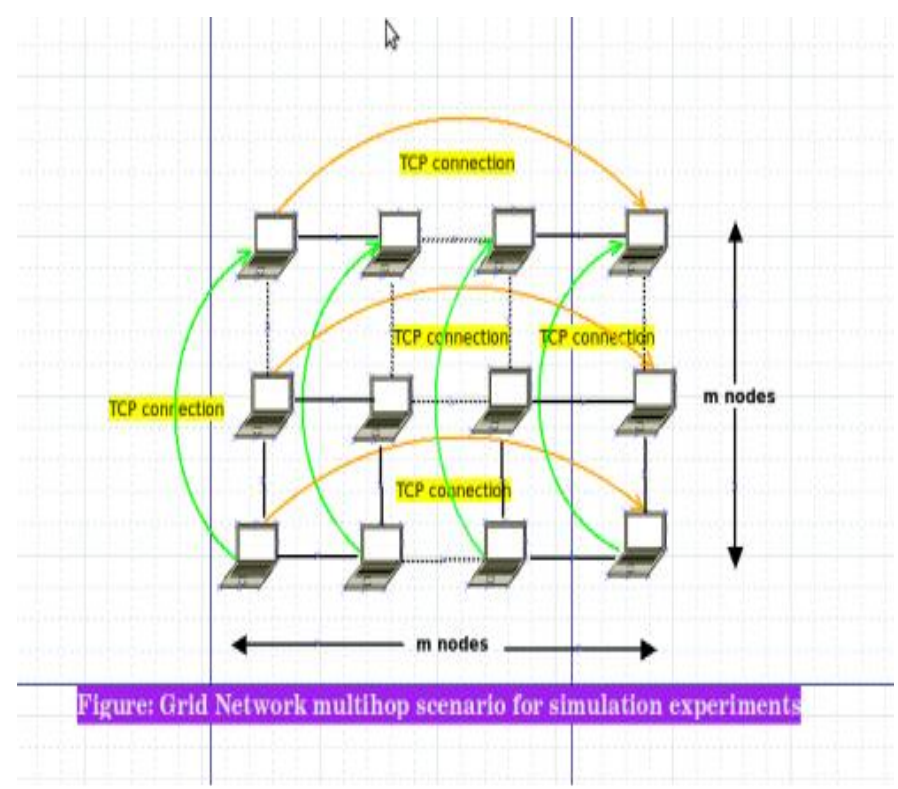

Fig. 10. Grid Topology
In each row, a TCP connection is assumed to set up from the left end node to the right end, and similarly, in each column, a TCP connection is assumed to set up from the bottom end node to the top end node. The histogram of average throughput of grid network shows that, TCP Costco Reno gives good average throughput then all TCP -variant except Reno.

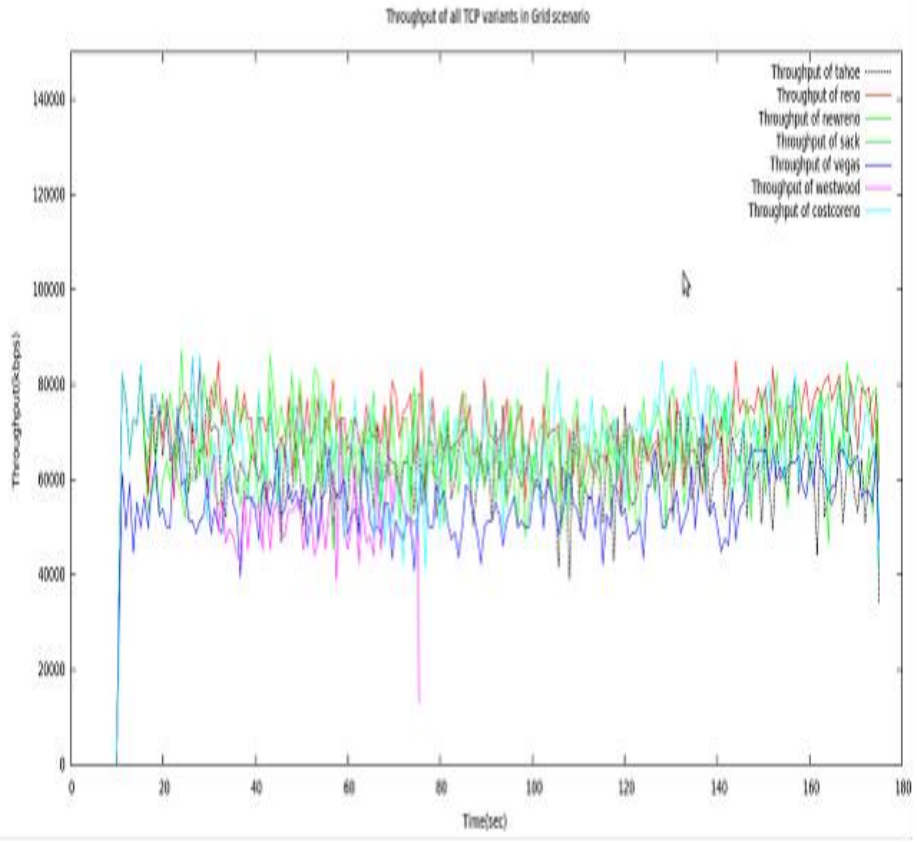

Fig. 11. Throughput plots of all TCP variant in grid networks

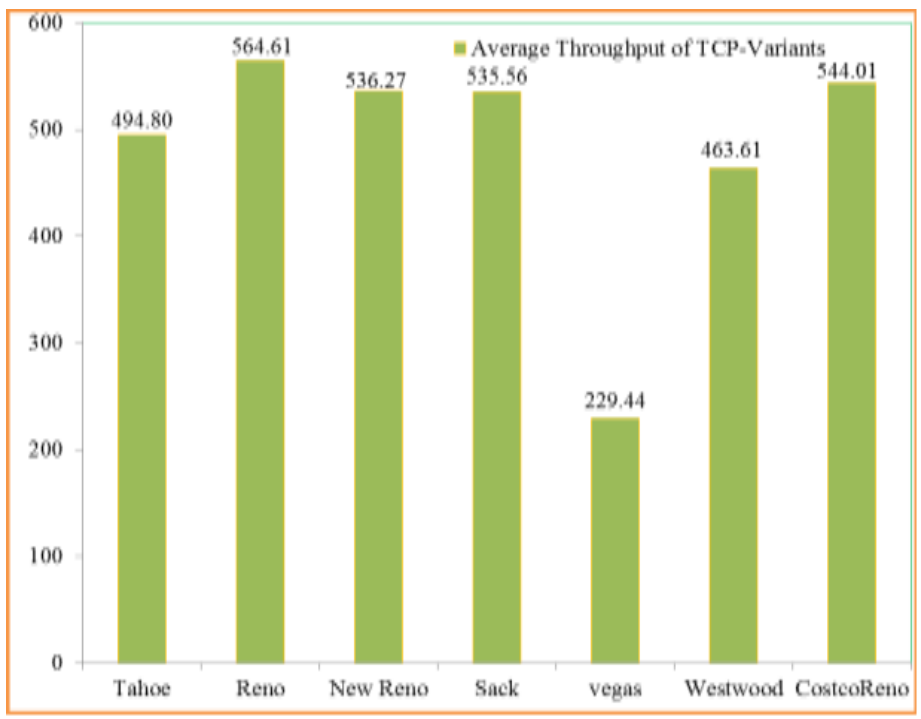

Fig. 12. Average Throughput of TCP Variants in Grid Networks 
TABLE III. Average Value Of Average Throughrut In All Scenario Of All TcP-Variant

\begin{tabular}{|c|c|c|c|c|c|c|c|}
\hline $\begin{array}{l}\text { Average of } \\
\text { average } \\
\text { Throughput }\end{array}$ & $\begin{array}{l}\text { Network } \\
\text { Scenario } \\
\text { TCP } \\
\text { Variants }\end{array}$ & $\begin{array}{c}\text { Congestion } \\
\text { Scenario }\end{array}$ & $\begin{array}{c}\text { Interference } \\
\text { Scenario }\end{array}$ & $\begin{array}{l}\text { Link Failure } \\
\text { Scenario }\end{array}$ & Signal Loss & $\begin{array}{l}\text { Chain } \\
\text { Network } \\
\text { Scenario }\end{array}$ & $\begin{array}{c}\text { Grid } \\
\text { Network } \\
\text { Scenario }\end{array}$ \\
\hline C. 429.94 & Tahoe & D. 524.50 & E. 678.7 & F. 497.07 & G. 156.93 & H. 227.68 & I. 494.80 \\
\hline J. 440.855 & Reno & K. 525.63 & L. 679.4 & M. 494.57 & N. 156.24 & O. 227.68 & P. 564.61 \\
\hline Q. 434.271 & $\begin{array}{l}\text { New } \\
\text { Reno }\end{array}$ & R. 508.07 & S. 679.62 & T. 497.03 & U. 156.96 & V. 227.68 & W. 536.27 \\
\hline$X .433 .276$ & Sack & Y. 512.86 & Z. $\quad 679.77$ & ${ }_{89}{ }_{89} 496$. & $\begin{array}{ll}B B . & 146 .\end{array}$ & $\underset{68}{C C .} 227$. & $\begin{array}{c}D D . \\
35.56\end{array}$ \\
\hline${ }_{38}{ }_{38} 217.1$ & Vegas & $\begin{array}{ll}F F . & 271 .\end{array}$ & $\begin{array}{cc}G G . & 348 . \\
5 & \end{array}$ & $\begin{array}{l}H H . \\
84\end{array}$ & II. 84.65 & JJ. 116.44 & $\begin{array}{cc}K K . & 2 \\
29.44\end{array}$ \\
\hline LL. 427.348 & $\begin{array}{c}\text { Westwoo } \\
\mathrm{d}\end{array}$ & $\begin{array}{cc}M{ }_{66} & 540 .\end{array}$ & $\begin{array}{ll}N N . & 678 . \\
88 & \end{array}$ & $\begin{array}{ll}\text { OO. } & 497 .\end{array}$ & ${ }_{19}^{P P .} 156$. & $\underset{68}{Q Q .} 227$. & $R R . \quad 4$ \\
\hline SS. 437.501 & $\begin{array}{l}\text { Costco } \\
\text { Reno }\end{array}$ & TT.515.48 & $\begin{array}{ll}U U . & 679 .\end{array}$ & $V V .{ }_{18} 497$. & $\begin{array}{cc}W W . & 161 . \\
04 & \end{array}$ & $\underset{68}{X X}$ & $Y Y .544 .01$ \\
\hline
\end{tabular}

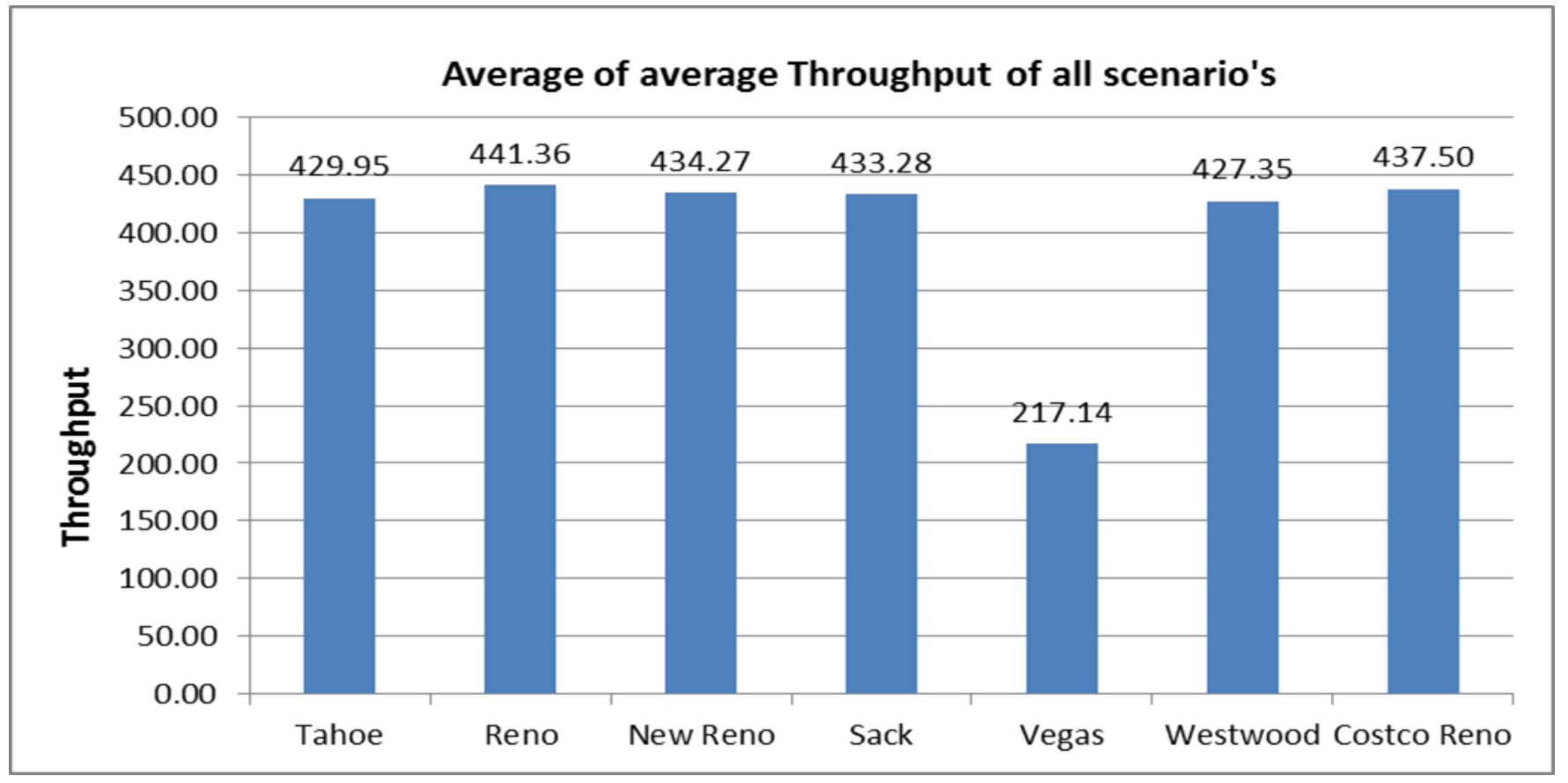

Fig. 13. Average of average Throughput of TCP Variants in all Network Scenario's 


\section{ACKNOWLEDGMENT}

I am deeply thankful to my M. Tech. Mentor Dr. Vijay Raisinghani who has boosted morale, confidence and taught how to do research, my friend Prof. Sanjay Sange for constantly encouragement, motivation and helps for best quality work and at last but not least to my father who has shown such a glorious and beautiful days days.

\section{CONCLUSION}

This paper presents a thorough literature survey of TCPWestwood and TCP-WELCOME along with its comparative analysis study and problems encountered in MANETs. As in case of wireless networks, performance of TCP degrades because of its inability to handle wireless channel errors. The proposed article placed special emphasis on those TCPVariants that preserve end-to-end semantic and most successful over mobile ad Hoc Network. This article Proposed Bandwidth estimation based TCP-Costco Reno, a new variant which is the modification of TCP-Westwood. With reference to the experimental results and data analysis, it shows that, TCP-Costco Reno handles much efficiently the wireless channel errors (signal loss and interference). Hence it improves overall throughput without degrading TCP performance in other scenarios and suitable for mobile ad hoc networks to avoid over or under estimation of BWE value. It outperforms in different types of wireless channel errors such as signal loss, link failure and interference and gives improved performance in grid network. Simulated result also indicates that, Average of average throughput of TCP Costco Reno in all scenarios is better than all other variants.

\section{REFERENCES}

[1] J. Li, C. Blake, D.S.J. De Couto, H.I. Lee, and R. Morris, "Capacity of Ad Hoc Wireless Networks,' Proc. ACM MobiCom '01, July 2001.

[2] Z. Fu, P. Zerfos, H. Luo, S. Lu, L. Zhang, and M. Gerla, "The Impact of Multi hop Wireless Channel on TCP Throughput and Loss," Proc. INFOCOM '03, Apr. 2003.

[3] K. Chen, Y. Xue, and K. Nahrstedt, "On Setting TCP's Congestion Window Limit in Mobile Ad Hoc Networks," Proc. IEEE Int'l Conf. Comm. (ICC '03), May 2003.

[4] A. Seddik-Ghaleb, Y. Ghamri-Doudane, and S. M. Senouci, "TCP WELCOME TCP Variant for Wireless Environment, Link losses, and
COngestion packet loss ModEls," in First International Communication Systems and Networks and Workshops, COMSNETS 2009.

[5] Bhaskar Sardar, Debashis Saha, "A Survey of TCP Enhancements for Last-Hop Wireless Networks" in IEEE Communications Surveys \& Tutorials, 2006 3rd Quarter 2006,Volume 8, No 3.

[6] Haifa Touati, Ilhem Lengliz, Farouk Kamoun, "TCP Adaptive RTO to Improve TCP performance in mobile ad hoc networks," in The Sixth Annual Mediterranean Ad Hoc Networking WorkShop, Corfu, Greece, June 12-15, 2007.

[7] Network Simulator Ns2, http://www.isi.edu/nsnam/ns.

[8] H. M. El-Sayed, "Performance Evaluation of Tcp in Mobile Ad-Hoc Networks," in the Second International Conference on Innovations in Information Technology (IIT) 2005.

[9] Adib M.Monzer Habbal, "Loss Detection and Recovery Techniques for TCP in Mobile Ad Hoc Network" in Second International Conference on Network Applications, Protocols and Services, 2010.

[10] Mario Gerla, M. Y. Sanadidi, Ren Wang, and Andrea Zanella, "TCP Westwood: Congestion Window Control Using Bandwidth Estimation," UCLA Computer Science Department, 0-7803-7206-9/ 2001

[11] Ahmad Al Hanbali, Eitan Altman, And Philippe Nain, Inria Sophia Antiplolis France," A SURVEY OF TCP OVER AD HOC NETWORKS", IEEE Communications Surveys \& Tutorials ,Third Quarter 2005.

\section{AUTHORS PROFILE}

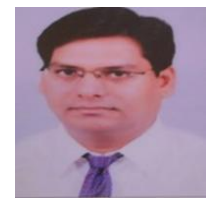

Prakash B. Khelage received his B.E. in Electronics and Telecommunication Engineering from Dr. Babasaheb Ambedkar Marathwada University Aurangabad, M.Tech in information Technology from NMIMS University Mumbai, Maharastra, India. He is currently working as Assistant Professor with UMIT, SNDT Women's University. He has 13 years of experience in industrial as well as educational field; His research interest includes Ad Hoc Networks, Mobile Computing, Wireless Networks, Co-operative Communication Networks and Network Security. He has also interest in Computer Architecture design, Cloud Computing and Data Mining.

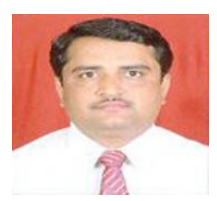

Uttam D. Kolekar received his B.E. in Electronics and Telecommunication Engineering, M.E. in Electronics from Shivaji University, Kolhapur and Awarded Ph. D. in electronics from Bharati Vidhyapith Pune, Maharastra, India. He is currently working as Principal with Smt. Indira Gandhi College of Engineering, Mumbai University. He has more than 20 years of experience in educational institution; His research interest includes Ad Hoc Networks, Mobile Computing, Wireless Networks, Neural Network and Co-operative Communication Networks. He has published over 30 National and International Jurnals \& conferences various papers accros India and other countries. 